\title{
A Diamagnetic Dititanium(III) Paddlewheel Complex With No Direct Metal-Metal Bond
}

Arjun Mendiratta, $\uparrow$ Christopher C. Cummins, ${ }^{*}, \dagger$ F. Albert Cotton*, Sergey A. Ibragimov,+ Carlos A. Murillo*, + and Dino Villagrán $\ddagger$

\section{Supporting Information}

For general synthetic considerations please see a recent representative publication. ${ }^{1}$ The amines $\mathrm{HN}\left(\mathrm{Bu}^{t}\right) \mathrm{Ar}^{2}$ and $\mathrm{HN}\left(\mathrm{Bu}^{t}\right) \mathrm{Ph}^{3}$ were prepared according to published procedures, as were the titanium(III) trisamides, $\operatorname{Ti}\left[\mathrm{N}\left(\mathrm{Bu}^{t}\right) \mathrm{Ar}\right]_{3}$ and $\mathrm{Ti}\left[\mathrm{N}\left(\mathrm{Bu}{ }^{t}\right) \mathrm{Ph}\right]_{3} \cdot{ }^{4} \mathrm{H}$ NMR spectra of 1a were collected at ambient temperature at MIT on a Varian INOVA-501 spectrometer at $500 \mathrm{MHz}$ and at various temperatures at TAMU on a Bruker VXR-300 spectrometer. Variable-temperature magnetic susceptibility measurements were made on a Quantum Design SQUID magnetometer MPMS-XL housed at Texas A\&M University. Data were collected from 2 to $300 \mathrm{~K}$ at a field of $1000 \mathrm{G}$, and the data were corrected empirically for diamagnetism of the sample and the holder.

Synthesis of $\mathbf{T i}_{2}\left[\mu_{2}-\mathrm{O}_{2} \mathrm{CN}\left(\mathrm{Bu}^{t}\right) \mathbf{A r}\right]_{4}\left(\mathrm{~N}\left(\mathrm{Bu}^{t}\right) \mathbf{A r}\right)_{2}$, 1a. In an $\mathrm{N}_{2}$-atmosphere glovebox, a $250 \mathrm{~mL}$ round-bottom flask with sidearm was charged with dark emerald green $\operatorname{Ti}\left[\mathrm{N}\left(\mathrm{Bu}^{t}\right) \mathrm{Ar}_{3}(696 \mathrm{mg}, 1.21 \mathrm{mmol})\right.$ and $\mathrm{Et}_{2} \mathrm{O}(30 \mathrm{~mL})$. The flask was fitted with a septum and placed briefly under reduced pressure prior to being sealed and removed from the glovebox. After cooling to $-40{ }^{\circ} \mathrm{C}$ using an $\mathrm{MeCN} / \mathrm{N}_{2}(1)$ slush bath, $\mathrm{CO}_{2}(100 \mathrm{~mL}$, 4.46 mmol, 3.7 equiv) was added via syringe. The emerald green solution was stirred for $2.5 \mathrm{~h}$, during which time the bath temperature rose to $0^{\circ} \mathrm{C}$ and a lime green precipitate appeared. The bath was removed and the mixture permitted to warm to ambient temperature (ca. $25^{\circ} \mathrm{C}$ ). The solvent was removed in vacuo. In the glove box, the lime 
green powder was slurried in pentane $(10 \mathrm{~mL})$, collected by filtration, and further washed with pentane $(5 \mathrm{~mL})$ and $\mathrm{Et}_{2} \mathrm{O}(5 \mathrm{~mL})$. The solid was dried under vacuum to yield $\mathrm{Ti}_{2}\left[\mu_{2^{-}}\right.$ $\left.\mathrm{O}_{2} \mathrm{CN}\left(\mathrm{Bu}^{t}\right) \mathrm{Ar}\right]_{4}\left(\mathrm{~N}\left(\mathrm{Bu}^{t}\right) \mathrm{Ar}\right)_{2}(640 \mathrm{mg}, 0.968 \mathrm{mmol}, 80 \%) .{ }^{1} \mathrm{H} \mathrm{NMR}\left(500 \mathrm{MHz}, \mathrm{C}_{6} \mathrm{D}_{6}\right.$, at $25^{\circ} \mathrm{C}: \delta(\mathrm{ppm})=1.48\left(\mathrm{~s}, 9 \mathrm{H}\right.$, amide $\left.\mathrm{CMe}_{3}\right)$, ca. $1.6\left(\mathrm{br} \mathrm{s}, 18 \mathrm{H}\right.$, carbamate $\left.\mathrm{CMe} e_{3}\right)$, ca. 2.3 (br s, $12 \mathrm{H}$, carbamate $\left.\mathrm{C}_{6} \mathrm{H}_{3} M e_{2}\right), 2.46\left(\mathrm{~s}, 6 \mathrm{H}\right.$, amide $\left.\mathrm{C}_{6} \mathrm{H}_{3} M e_{2}\right)$, ca. 6.4 (v br, $4 \mathrm{H}$, carbamate ortho), 6.75 (s, 2H, amide ortho), 6.79 (s, 1H, amide para), ca. 7.6 (v br, 2H, carbamate para) ppm. Anal. Calcd. for $\mathrm{C}_{76} \mathrm{H}_{108} \mathrm{~N}_{6} \mathrm{O}_{8} \mathrm{Ti}_{2}:$ C, 68.66; H, 8.19; N, 6.32. Found: C, 68.19; H, 7.93; N, 6.29.

Synthesis of $\mathrm{Ti}_{2}\left[\mu_{2}-\mathrm{O}_{2} \mathrm{CN}\left(\mathrm{Bu}^{t}\right) \mathbf{P h}\right]_{4}\left(\mathrm{~N}\left(\mathrm{Bu}^{t}\right) \mathbf{P h}\right)_{2}, \mathbf{1 b}$. This synthesis was carried out as described for 1a, substituting $\operatorname{Ti}\left[N\left(\mathrm{Bu}^{t}\right) \mathrm{Ph}\right]_{3}$ for $\operatorname{Ti}\left[\mathrm{N}\left(\mathrm{Bu}^{t}\right) \mathrm{Ar}\right)_{3}$. Crystals for the X-ray diffraction study were grown from a $\mathrm{THF} / \mathrm{Et}_{2} \mathrm{O}$ solvent mixture. The color and physical properties are similar to those of $\mathbf{1 a}$, as is the ${ }^{1} \mathrm{H}$ NMR, which shows some broad bands at room temperature because of the molecule being fluxional. ${ }^{1} \mathrm{H}$ NMR $\left(500 \mathrm{MHz}, \mathrm{C}_{6} \mathrm{D}_{6}\right.$, at $\left.25{ }^{\circ} \mathrm{C}: \delta, \quad \mathrm{ppm}\right)=1.26\left(\mathrm{~s}, \quad 9 \mathrm{H}, \quad\right.$ amide $\left.\mathrm{CMe}_{3}\right) ; 1.4 \quad($ br $\mathrm{s}, \quad 18 \mathrm{H}$, carboxamide $\mathrm{CMe}_{3}$ ); 6.43 (br s, $4 \mathrm{H}$ carboxamide ortho); 6.85 (br s, $2 \mathrm{H}$, amide ortho); 7.03 (two overlapping peaks, 4H, carboxamide para and amide ortho); 7.18 (t, 1H, amide para); 7.4 (br s, 4H, carboxamide meta). The aryl peak assignments are tentative.

\section{X-ray structure determination for $\operatorname{Ti}_{2}\left(\mu_{2}-\mathrm{O}_{2} \mathbf{C N}\left[\mathbf{B u} u^{t}\right] \mathbf{P h}\right)_{4}\left(\mathbf{N}\left[\mathbf{B u}{ }^{t}\right] \mathbf{P h}\right)_{2}$. Data was} collected on a Siemens Platform three-circle diffractometer equipped with a CCD detector and outfitted with a low-temperature, nitrogen-stream aperture. The structure was solved using direct methods, in conjunction with standard difference Fourier techniques and refined by full-matrix least-squares procedures. A summary of the 
crystallographic data is provided in Table 1. An empirical absorption correction (SADABS) was applied to the diffraction data. All non-hydrogen atoms were refined anisotropically. Unless otherwise specified, all hydrogen atoms were placed in idealized positions and refined isotropically. All software used for diffraction data processing and crystal-structure solution and refinement are contained in the SAINT+ (v6.45) and SHELXTL (v6.14) program suites, respectively (G. Sheldrick, Bruker AXS, Madison, WI).

Computational Details. DFT calculations were performed using the hybrid B3LYP ${ }^{5}$ functional as implemented in the Gaussian 03 (revision C.02) suite. ${ }^{6}$ A small effective core potential by Hay and Wadt along its corresponding double- $\zeta$ basis set (LANL2DZ) ${ }^{7}$ was utilized for the Ti atoms. Double- $\zeta$ quality basis sets (D95) ${ }^{8}$ were used on carbon, nitrogen, and hydrogen atoms. The geometries of all the models were optimized, and found to be local minima in a potential energy surface by performing frequency calculations and observing a lack of imaginary vibrations. The calculated B3LYP total energy of the high spin (H.S.) and broken-symmetry (B.S.) models in hartrees are -1206.5893 and -1206.5923 , respectively. The calculated B3LYP energy for the singlet model in hartrees is -1206.5596 . The calculated $\left\langle S^{2}\right\rangle$ values for the H.S. and B.S. models are 2.00 and 0.957 , respectively. All calculations were performed on an Origin 2000 32-processor SGI computer located at the Texas A\&M supercomputing facility.

Table 1. Crystal Data for $\mathrm{Ti}_{2}\left[\mu_{2}-\mathrm{O}_{2} \mathrm{CN}\left(\mathrm{Bu}^{t}\right] \mathrm{Ph}\right]_{4}\left(\mathrm{~N}\left(\mathrm{Bu}^{\mathrm{t}}\right) \mathrm{Ph}\right)_{2} \cdot \mathrm{Et}_{2} \mathrm{O}, \mathbf{1 b}$

\begin{tabular}{l|l}
\hline Chemical formula & $\mathrm{C}_{68} \mathrm{H}_{93} \mathrm{~N}_{6} \mathrm{O}_{9} \mathrm{Ti}_{2}$ \\
$\mathrm{fw}$ & 1234.43 \\
Space group & $P \overline{\mathrm{1}}$ \\
$a, \AA$ & $11.6091(16)$ \\
$b, \AA$ & $12.8853(18)$ \\
$c, \AA$ & $24.358(3)$ \\
$\alpha, \operatorname{deg}$ & $104.509(4)$ \\
$\beta, \operatorname{deg}$ & $92.288(4)$ \\
\hline
\end{tabular}




\begin{tabular}{l|l}
\hline$\gamma, \mathrm{deg}$ & $101.522(5)$ \\
$V, \AA^{3}$ & $3432.8(8)$ \\
$Z$ & 2 \\
$D, \mathrm{~g} / \mathrm{cm}^{3}$ & 1.194 \\
$\mu\left(\mathrm{Mo} \mathrm{K}_{\alpha}\right), \mathrm{mm}^{-1}$ & 0.290 \\
$\mathrm{~T}, \mathrm{~K}$ & 193 \\
$F(000)$ & 1318 \\
$\mathrm{GoF}\left(F^{2}\right)$ & 1.002 \\
$R(F), \%$ & 0.0574 \\
$w R(F), \%$ & 0.1306 \\
\hline
\end{tabular}

\footnotetext{
${ }^{1}$ Stephens, F. H.; Johnson, M. J. A.; Cummins, C. C.; Kryatov, O. P.; Kryatov, S. V.; Rybak-Akimova, E. V.; McDonough, J. E.; Hoff, C. D. J. Am. Chem. Soc. 2005, 127, 15191-15200.

${ }^{2}$ Tsai, Y. C.; Stephens, F. H.; Meyer, K.; Mendiratta, A.; Gheorghiu, M. D.; Cummins, C. C.

Organometallics 2003, 22, 2902-2913.

${ }^{3}$ Johnson, A. R.; Cummins, C. C.; Gambarotta, S. Inorg. Synth. 1998, 32, 123-132.

${ }^{4}$ Peters, J. C.; Johnson, A. R.; Odom, A. L.; Wanandi, P. W.; Davis, W. M.; Cummins, C. C. J. Am. Chem. Soc. 1996, 118, 10175-10188.

5 (a) Becke, A. D. Phys. Rev. A 1988, 38, 3098-3100. (b) Becke, A. D. J. Chem. Phys. 1993, 98, 1372-
}

1377. (c) Becke, A. D. J. Chem. Phys. 1993, 98, 5648-5652. (c) Lee, C.; Yang, W.; Parr, R. G. Phys. Rev. B 1988, 37, 785-789.

${ }^{6}$ Frisch, M. J.; Trucks, G. W.; Schlegel, H. B.; Scuseria, G. E.; Robb, M. A.; Cheeseman, J. R.; Montgomery, J. A., Jr.; Vreven, T.; Kudin, K. N.; Burant, J. C.; Millam, J. M.; Iyengar, S. S.; Tomasi, J.; Barone, V.; Mennucci, B.; Cossi, M.; Scalmani, G.; Rega, N.; Petersson, G. A.; Nakatsuji, H.; Hada, M.; Ehara, M.; Toyota, K.; Fukuda, R.; Hasegawa, J.; Ishida, M.; Nakajima, T.; Honda, Y.; Kitao, O.; Nakai, H.; Klene, M.; Li, X.; Knox, J. E.; Hratchian, H. P.; Cross, J. B.; Bakken, V.; Adamo, C.; Jaramillo, J.; Gomperts, R.; Stratmann, R. E.; Yazyev, O.; Austin, A. J.; Cammi, R.; Pomelli, C.; Ochterski, J. W.; Ayala, P. Y.; Morokuma, K.; Voth, G. A.; Salvador, P.; Dannenberg, J. J.; Zakrzewski, V. G.; Dapprich, S.; Daniels, A. D.; Strain, M. C.; Farkas, O.; Malick, D. K.; Rabuck, A. D.; Raghavachari, K.; Foresman, J. B.; Ortiz, J. V.; Cui, Q.; Baboul, A. G.; Clifford, S.; Cioslowski, J.; Stefanov, B. B.; Liu, G.; Liashenko, A.; Piskorz, P.; Komaromi, I.; Martin, R. L.; Fox, D. J.; Keith, T.; Al-Laham, M. A.; Peng, C. Y.; Nanayakkara, A.; Challacombe, M.; Gill, P. M. W.; Johnson, B.; Chen, W.; Wong, M. W.; Gonzalez, C.; Pople, J. A. Gaussian 03, revision C.02; Gaussian, Inc.: Wallingford, CT, 2004.

${ }^{7}$ (a) Wadt, W. R.; Hay, P. J. J. Chem. Phys. 1985, 82, 284-298. (b) Hay, P. J.; Wadt, W. R. J. Chem. Phys. 1985, 82, 299-310.

${ }^{8}$ Dunning, T. H.; Hay, P. J. In Modern Theoretical Chemistry. 3. Methods of Electronic Structure Theory; Schaefer, H. F., III, Ed.; Plenum Press: New York, 1977; pp 1-28. 


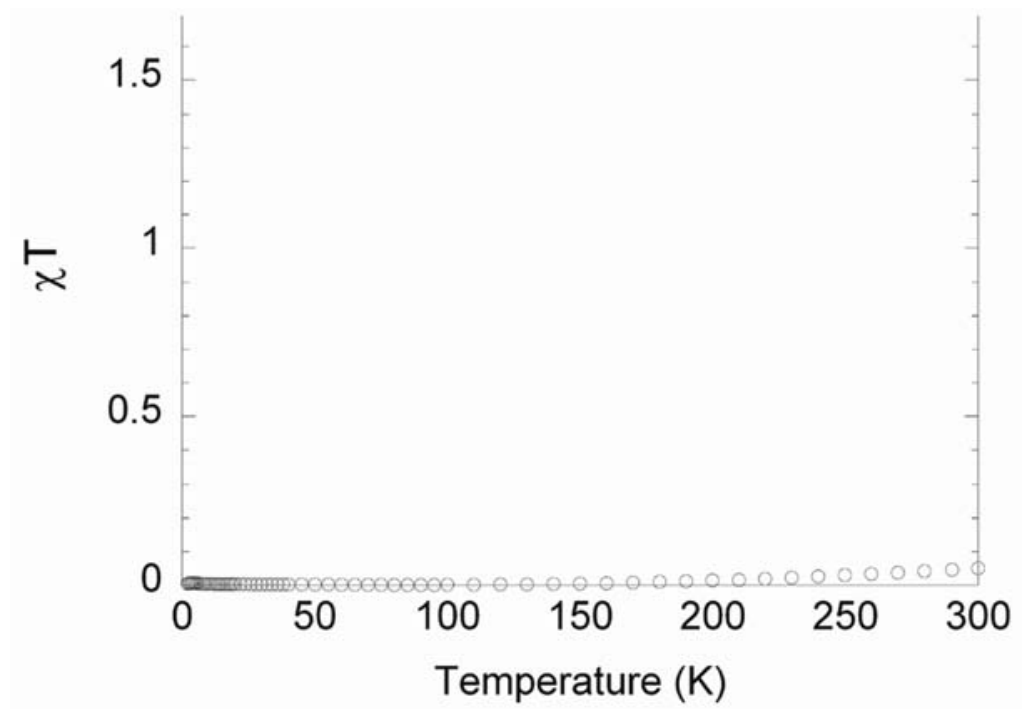

Fig S1. Plot of $\chi T$ vs $T$ for $1 \mathrm{a}$ in the solid state. 